\title{
Effects of different milk substitutes on the pasting, rheological and textural properties of puddings
}

\author{
Cristina Alamprese and Manuela Mariotti \\ Dipartimento di Scienze e Tecnologie Alimentari e Microbiologiche (DiSTAM), University of Milan, Via \\ Celoria 2, 20133 Milano, Italy
}

\begin{abstract}
Puddings are usually milk protein-based starch pastes, but the use of milk substitutes in their preparation could be interesting for people suffering of lactose intolerance and milk allergies. The study investigated the pasting, rheological, and textural behaviors of two commercial pudding powders ( $A$ and B) dissolved in rice drink, light soy drink, partially skimmed milk or deionized water. Samples were evaluated during both the preparation (heating and cooling phases) and the storage over a 3 day refrigeration period. Generally, the properties of soy drink-based puddings were similar to those of the reference samples, while the characteristics of rice drink-based products were comparable to those of the water systems. The two commercial powders, apparently similar in formulation, exhibited dramatic differences both in the pasting and rheological behaviors. The gel settling took place mainly during the heating phase for sample $A$ and during the cooling period for sample $B$, probably due to the different starch and carrageenan types present in the powders. This aspect could be also responsible for the different textural behavior of the puddings during the storage period: powder $B$ dissolved in rice drink and water gave stiffer products in comparison with powder A dispersed in the same media.
\end{abstract}

Keywords: Milk substitutes, pasting properties, pudding, rheology, texture.

\section{INTRODUCTION}

Commercial pudding powders are usually composed of starch, hydrocolloids, sugars, colorings and aromas and they are intended to be dissolved in milk [1]. In fact, puddings are generally milk proteinbased starch pastes, with the typical texture of a semisolid food [2]. Although the structure and the rheological behavior of these products are known to be strongly influenced by the concentrations and particular characteristics of each ingredient and by the cross-interactions among them [1], all these aspects have been little studied. In particular, few works deal with the use of milk substitutes in pudding preparation, interesting for people suffering of lactose intolerance and milk allergies.

The aim of the study was to investigate the pasting, rheological, and textural behaviors of two commercial pudding powders dissolved in rice drink and light soy drink For each powder, a reference sample obtained with partially skimmed milk and a model system containing water were also studied.

\section{MATERIALS AND METHODS}

\subsection{Materials and sample preparation}

Two different commercial powders for vanilla pudding were used ( $A$ and $B$ ), both containing starch, glucose, carrageenans, salt, colorings, and aromas. Powders $(35 \mathrm{~g})$, added with sucrose $(45 \mathrm{~g})$, were slowly dispersed in $500 \mathrm{~mL}$ rice drink (samples A-R and B-R), light soy drink (samples A-S and B$S$ ), partially skimmed milk (samples $A-M$ and $B-M$ ), or deionized water (samples A-W and B-W). For the viscoamylographic test, $100 \mathrm{~mL}$ dispersion were used. For rheological analyses, dispersions were stirred for $20 \mathrm{~min}$ at room temperature and for 10 min in a water bath at $60^{\circ} \mathrm{C}$ before sampling. For textural analyses, the dispersions were placed in a water bath at $100^{\circ} \mathrm{C}$ and continuously stirred until the mixture temperature reached $85^{\circ} \mathrm{C}$. This final temperature was maintained $2 \mathrm{~min}$, then the puddings were distributed in polythene hinged-lid containers ( $30 \mathrm{~g}$ each) and placed in a cold room at $4 \pm 1^{\circ} \mathrm{C}$.

\subsection{Pasting behavior}

The pasting behavior was assessed by a MicroVisco-Amylograph (Brabender OHG, Duisburg, Germany). Pudding dispersions were evaluated under constant conditions (stirring: $250 \mathrm{~min}^{-1}$, sensitivity: $300 \mathrm{~cm} \cdot \mathrm{gf}$ ) according to the following time-temperature profile: heating from $25^{\circ} \mathrm{C}$ to $95^{\circ} \mathrm{C}$ $\left(3^{\circ} \mathrm{C} / \mathrm{min}\right)$; maintenance at $95^{\circ} \mathrm{C}$ for $2 \mathrm{~min}$; cooling to $25^{\circ} \mathrm{C}\left(3^{\circ} \mathrm{C} / \mathrm{min}\right)$. Viscosity and temperature data were recorded over time. Each analysis was done at least in duplicate on freshly prepared samples.

\subsection{Rheological behavior}

The rheological behavior of samples was assessed by temperature sweep tests carried out using a Physica MCR 300 rheometer (Anton Paar GmbH, Graz, Austria) equipped with coaxial cylinders (CC27). A $200 \mathrm{~s}^{-1}$ shear rate was used, measuring the temperature dependence of apparent viscosity ( $\eta$, Pa s). The following time-temperature profile was applied: heating $\left(3^{\circ} \mathrm{C} / \mathrm{min}\right)$ from $60^{\circ} \mathrm{C}$ up to $95^{\circ} \mathrm{C}$; maintenance at $95^{\circ} \mathrm{C}$ for $2 \mathrm{~min}$; cooling to $5^{\circ} \mathrm{C}$ $\left(3^{\circ} \mathrm{C} / \mathrm{min}\right)$. Determinations were performed at least in duplicate on freshly prepared samples.

\subsection{Textural characteristics}

Texture of puddings was assessed by an Instron 
Universal Testing Machine (Mod. 3365, Instron Ltd., High Wycombe, UK) on $30 \mathrm{~g}$ samples during cooling from $85^{\circ} \mathrm{C}$ to $4^{\circ} \mathrm{C}$ (after 15, 30, 60, $120 \mathrm{~min}$ ) and during storage for $16,24,48$, and $72 \mathrm{~h}$ at $4^{\circ} \mathrm{C}$. Penetration tests were carried out at a constant rate (50 $\mathrm{mm} / \mathrm{min}$ ), using a $27 \mathrm{~mm}$ diameter plate connected to a $100 \mathrm{~N}$ load cell. Penetration was continued down to $30 \%$ of the initial height of the sample. Results, expressed as the load (N) at $30 \%$ strain, were the average of four measurements. Each pudding sample was prepared at least in duplicate.

\section{RESULTS AND DISCUSSION}

\subsection{Pasting and rheological behaviors}

Pasting and rheological behaviors of the different dispersions are shown in Fig. 1. The two commercial powders, although apparently similar in formulation, behaved differently: the gel settling took place mainly during the heating phase for sample $A$ and during the cooling period for sample B.

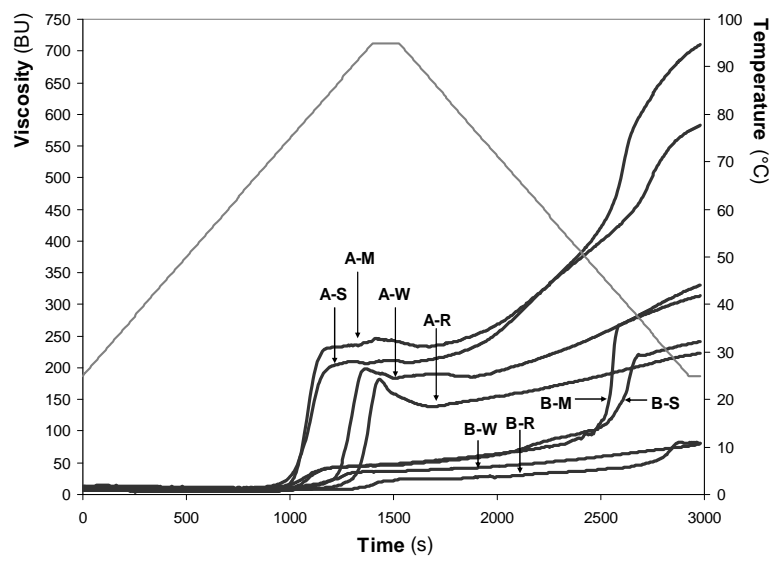

Figure 1: Pasting behavior of pudding samples.

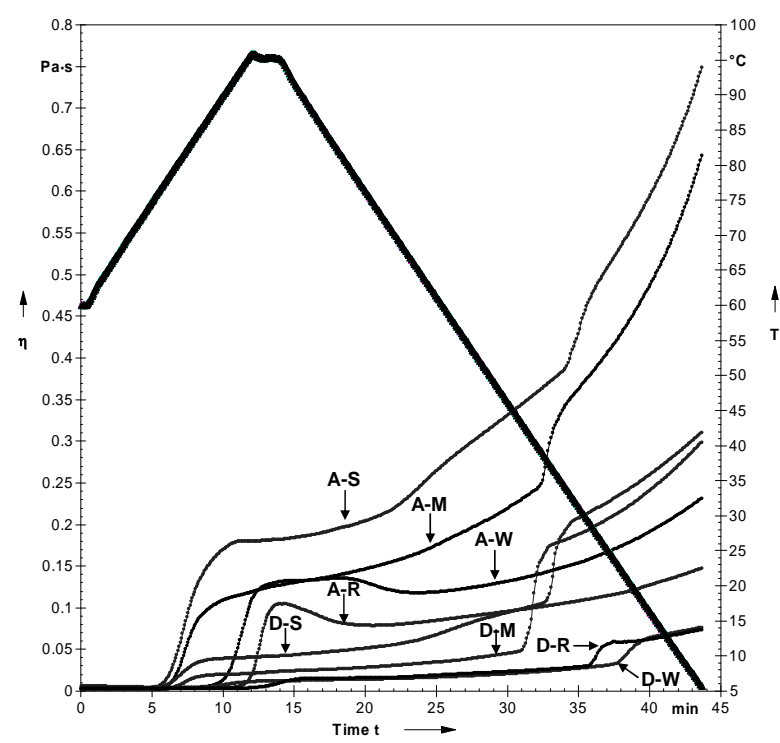

Figure 2: Apparent viscosity $(\eta)$ of pudding samples measured by temperature ( $T$ ) sweep test.
As regards the effects of the four different media, the properties of soy drink-based puddings were similar to those of milk-based samples, while the characteristics of rice drink-based products were comparable to those of the water systems.

\subsection{Textural characteristics}

Fig. 3 shows texture modifications of puddings during cooling and storage at $4^{\circ} \mathrm{C}$. Samples reached almost the maximum firmness just after $2 \mathrm{~h}$ of cooling. Both sample A and $B$ reached the highest load values when dispersed in milk, being sample $B$ $M$ less firm than A-M. A good structuring was also obtained using soy drink. On the contrary, rice drink proved not to be a good dispersing medium for pudding production, resulting in products with poor structure, comparable to those obtained with water. It is interesting to notice that powder B dispersed in rice drink and water produced puddings stiffer than those obtained from powder $A$.

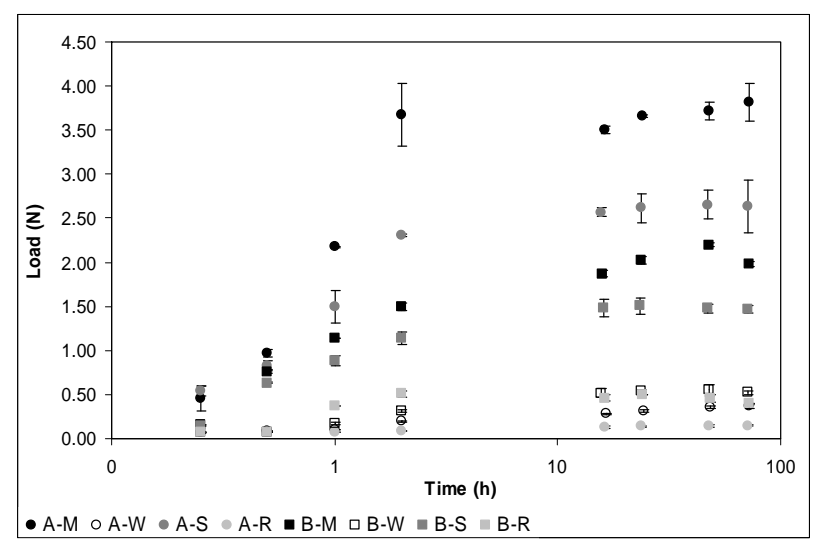

Figure 3: Load at $30 \%$ strain of pudding samples during cooling and storage at $4^{\circ} \mathrm{C}$.

\subsection{Conclusions}

Commercial pudding powders, even if apparently similar in formulation, could result in final products with large structural differences. This aspect could be ascribed to different types and quantity of starch and carrageenans present in the formulation. Moreover, even if a large number of milk substitutes is available on the market, they can not be indifferently used for pudding production as the optimal texture of the final product is not always achieved. For these reasons, the mechanisms behind the observed phenomena should be studied more in depth.

\section{REFERENCES}

[1] Vélez-Ruiz J, Hernando I, Gonzàlez-Tomàs L, PérezMunuera I, Quiles A, Tàrrega A, Lluch MA, Costell E: Rheology and Microstructure of Custard Model Systems with Cross-linked Waxy Maize Starch, Flavour Fragr. J. 21 (2006) 30-36.

[2] Lim HS, Narsimhan G: Pasting and Rheological Behavior of Soy Protein-based Pudding, LWT 29 (2006) 343-349. 